\title{
Achieving a running cost saving with a cabinet refrigerator incorporating a Phase Change Material by the scheduling optimisation of its cyclic operations
}

\author{
Angelo Maiorinoa,†, Manuel Gesù Del Ducaa , Adrián Mota-Babilonib, Ciro Aprea ${ }^{a}$ \\ a Department of Industrial Engineering, University of Salerno, Via Giovanni Paolo II 132, \\ 84084, Fisciano (SA), Italy \\ ${ }^{b}$ ISTENER Research Group, Department of Mechanical Engineering and Construction, \\ Campus de Riu Sec s/n, Universitat Jaume I, E-12071 Castelló de la Plana, Spain
}

\begin{abstract}
Phase Change Materials (PCMs) can absorb and release a high amount of energy, and therefore, can be used for shifting electricity demand in some household appliances. Indeed, the use of PCMs in a cabinet refrigerator leads to increase its OFF time and flexibility, and moves the energy consumption to other periods for a reduction of the peak energy demand. The higher flexibility of the refrigerator with PCM can be exploited to reduce its running cost considering that many countries adopted Time-of-Use (TOU) tariffs, in which the electricity cost is based on the time of energy use over a day and a week. Hence, this work proposes an algorithm based on Simulated Annealing (SA) method to identify the optimal working scheduling of a refrigerator with PCM in order to reduce its running costs. Experimental results have been used as inputs for the algorithm, and nine 2-TOU and three 3-TOU electricity tariffs from different European countries have been selected to test the methodology. The possibility to achieve running cost savings for various case studies has been proven. Furthermore, higher the difference between the peak and off-peak electricity cost, greater the economic benefits reached by the proposed method.
\end{abstract}

Keywords: Optimisation, Phase Change Material (PCM), Cost saving, Cooling, Time-Of-Use (TOU), Peak shifting

\footnotetext{
* Corresponding author: Tel. +39(0) 89 964105; fax +39089 964037; 


\section{HIGHLIGHTS}

- Phase Change Materials (PCMs) enhanced the operating flexibility of a cabinet refrigerator

- Experimental data were used to simulate cyclic operations of the system

- A hysteresis scheduling optimisation was performed by Simulated Annealing (SA)

- Running cost savings were obtained for different case studies

- The off-peak vs. peak cost ratio defines the amount of the achievable saving 


\section{Nomenclature}

Symbols
c
energy cost $\left[€ \mathrm{kWh}^{-1}\right]$
E
energy variable in the SA method [/]
EC
energy consumption [Wh]
$\mathrm{H}$
time horizon $[\mathrm{s}]$
n
number of scheduled hysteresis [/]
$\mathrm{P}$
average electric power [W]
S
state of the compressor [/]
st
number of steps at the same temperature in the SA method [/]
$\mathrm{T}$
temperature variable of the SA method [/]

Greek symbols

$\alpha$

$\Delta$

$\delta$

$\tau$

\section{Subscripts}

0

d

el

end

$\exp$

$\mathrm{H}$

i

$\mathrm{j}$

r

set

W cooling rate in the SA method [/]

hysteresis [K]

difference [/]

time [s]

initial

daily

electric

final

experimental

over the time horizon

time-step index [/]

hysteresis scheduling index [/]

running

pre-defined

weekly 
Superscripts

$\begin{array}{ll}\text { OFF } & \text { during OFF state } \\ \text { ON } & \text { during ON state } \\ \text { s } & \text { scheduled } \\ \text { ss } & \text { scheduled start }\end{array}$

Abbreviations

$\begin{array}{ll}\text { LDPE } & \text { low-density polyethylene } \\ \text { PCM } & \text { phase change material } \\ \text { SA } & \text { simulated annealing } \\ \text { TOU } & \text { time-of-use }\end{array}$




\section{Introduction}

The International Institute of Refrigeration estimates that the total number of refrigeration, airconditioning, and heat pump systems in operation worldwide in 2015 is approximately 3 billion. Moreover, the energy consumption of these devices is about $17 \%$ of the overall electricity used worldwide (Coulomb et al., 2015). The greenhouse gas emissions corresponding to this sector quantified in 2017 by the same organization accounts for approximately $7.8 \%$ worldwide (Coulomb et al., 2017).

Refrigerators and freezers work under ON/OFF operations the 24 hours of the day, being more time running during the day time, in which the peak consumption in the national electricity grids are concentrated. During this period, electricity production uses renewable energy but is complemented by fossil fuel power generation. Considering the example of Indonesia, McNeil et al. (2019) predicted that in 2030, the electricity demand at peak time will be mostly distributed among residential and commercial air conditioners and refrigerators, among other applications. Therefore, the translation of energy consumption appliances from the peak period (day) to the off-peak one (night) would be an environmentally friendly action.

Electricity consumption shifting to selected periods could be achieved by several strategies based on an increment of the flexibility of operation (Bergaentzlé et al., 2019). One of the most common methods to motivate the shift is the time-of-use (TOU) tariff. In this tariff, the price of the electricity depends on the hour of the day, day type (working day or weekend), and season. Yan et al. (2018) reviewed different price-driven demand response programs, and they proved that the application of the TOU tariff is the easiest to follow, and it can obtain a stable participating ratio despite its simplicity.

Refrigerators and freezers can be switched off for a certain period since their thermal characteristics allow them of certain inertia. However, without any modification or adaptive strategies, a refrigerator represents a typical static load without the possibility of being shiftable or sheddable (Wang et al., 2018). Different actions have been proposed to convert refrigerators and freezers in a shiftable electrical load. Schné et al. (2018) controlled the operation of a household refrigerator attending to the energy tariffs. This modification allows it to shift its operating periods to less expensive periods autonomously. The proposed control can save $9 \%$ of the energy bill and decrease the peak energy consumption by as much as $76 \%$. Zehir and Bagriyanik (2012) reduced the yearly energy cost of a refrigerator down to $11.4 \%$ by only considering these actions: control (shifting) of defrosting, multiple pricing, refrigerator control, higher thermal mass, better insulation, and additional payments. Dlamini and Cromieres (2012) studied three types of household applications control algorithms, and the most effective algorithm was coordinating the timing of frequent intermittent loads and moving these loads to off-peak times. They highlighted the potential of refrigerators to be adapted to them. They mentioned that a refrigerator could be coordinated across the other appliances to prevent simultaneous loading because it is interruptible for a maximum of 30 min. Still, there are different methods to increase their autonomy.

Phase change materials (PCMs) were proposed as a thermal storage system in several cold production applications, as domestic or commercial refrigerators, being placed to the evaporator, condenser, compartment, or inside the refrigeration circuit (Mastani Joybari et al., 2015). PCMs were used as a solution to uniformize the temperature inside the refrigerated compartment or to improve energy efficiency. However, a significant improvement achieved using PCMs is the increase of the autonomy (part of the operating cycle during which the compressor is switched off). Azzouz et al. 
(2009) were one of the first authors in highlighting the increase of continuous operation without electrical supply (ranging from 5 to $9 \mathrm{~h}$ with PCM and from 1 to $3 \mathrm{~h}$ without it). Elarem et al. (2017) proposed a configuration in which the PCM was attached to the heat exchanger, and it resulted in promising energy benefits. In the solution proposed by Cofré-Toledo et al. (2018), the power consumption, running time and operation cycles of the compressor only were deteriorated for the period that follows the defrosting operation. The addition of the PCM attached to the rack and the evaporator could enlarge the $\mathrm{ON}$ and $\mathrm{OFF}$ time of a cabinet refrigerator and keep the products inside the racks at a proper conservation temperature (Maiorino et al., 2019). At the sight of these and other positive results, Li et al. (2019) concluded that cold storage technology using PCMs has great potential; however, the present research is still in its initial stage and requires further development.

The use of PCM is a sustainable energy technique in refrigeration that can be used to reduce the electricity in peak hour consumption (Bista et al., 2018). Purohit y Dasgupta (2020) recommended that the establishment of suitable control system for optimum energy usage of PCM is important. Hovgaard et al. (2012) presented a power management scheme based on the thermal storage capabilities and predictions of varying loads and energy prices for reducing running costs of supermarket refrigeration systems. Barzin et al. (2015) combined a price-based control with PCM storage (eutectic solution of ammonium chloride) in the walls of a freezer, reaching a cost saving of $16.5 \%$ per day. Other researchers also considered the utilization of water (with small additions) as the PCM material. Wang et al. (2017) used water PCM in a cold storage house to absorb the cold thermal energy in the off-peak period, and the payback period of the prototype was about 4.1 years. Liu et al. (2017) added $3 \mathrm{~kg}$ of PCM (demineralized water with $1 \%$ superabsorbent polymer and $0.03 \%$ diatomite) in the air duct foam located opposite the evaporator and fan (fresh food compartment). The on-time ratio was reduced by $13.6 \%$, and the electricity consumption was increased by $4.9 \%$ during the OFF time. As this increase was seen during the off-peak period, cost savings could be achieved.

The PCM used in refrigeration systems is a possible solution to increase significantly the autonomy of these systems, which can be used to move compressor on period to the off-peak periods. Zehir and Bagriyanik (2012) highlighted that refrigerators are thermostatically controlled. Switching off many of them will make their working schedule undiversified. A few previous works optimised the energy cost of refrigeration systems with PCM according the variation of energy price, but they did not consider the possibility of modifying control parameters neither tariffs with different behaviour (SOMETHING ELSE???). This paper aims to investigate the possibility of using PCM in contact with the aluminium racks and bare tube evaporator of a cabinet refrigerator, controlling the ON/OFF hysteresis to adjust the operation of the system to an existing TOU tariff. Differently from previous analysis, a control parameter (the ON/OFF hysteresis) is included in this study. In detail, the proposed algorithm can provide the scheduling of the switches-on of the refrigeration system, as well as the hysteresis values for each working cycle. The hysteresis is a fundamental variable in these kinds of systems since it affects both the power absorbed by the compressor and the OFF time of the refrigeration system. This method can provide quick economic benefits making use of a real hardware implementation (inexpensive solution) as well as reducing the energy demand peaks at a global scale.

\section{Experimental methodology}

Experimental data measured from a commercial cabinet refrigerator are used to model the ON/OFF operations of the refrigerator with PCM working with different hysteresis. The single door 
refrigerator has a capacity of $520 \mathrm{l}$ divided into six racks, which has the $0.66 \mathrm{~m}^{2}$ evaporator attached to them and then, below it, the PCM. The air circulation within the cabinet is obtained by free convection but also includes twelve boxes with a capacity of $0.03 \mathrm{~m}^{3}$, and another two of $0.02 \mathrm{~m}^{3}$ (corresponding to the lower rack) in which the products are placed.

The PCM is contained in $24 \times 24 \mathrm{~cm}^{2}$ low-density polyethylene (LDPE) plastic boxes, filled each one with $3.0 \mathrm{~cm}^{3}$ (which corresponds to a thickness of $4.0 \mathrm{~mm}$ ) of chloride saltwater $(15 / 85$ in mass percentage), resulting in a freezing temperature of $-5^{\circ} \mathrm{C} .2 .4 \mathrm{~kg}$ of PCM covered each rack of the refrigerator, excluding the upper rack where only a layer of PCM $(1.2 \mathrm{~kg})$ was placed below it due to construction limitations. As a result, $15.6 \mathrm{~kg}$ of PCM were used in the experimental tests. For further information about the distribution of the PCM, please refer to Maiorino et al. (2019). The following conditions have been set for evaluating the behaviour of the system in terms of ON/OFF time, and power absorbed by the compressor:

- Set-point temperature of $2{ }^{\circ} \mathrm{C}$ with three temperature hysteresis, $\pm 1, \pm 2$ and $\pm 3 \mathrm{~K}$;

- Ambient temperature of $25 \pm 0.5^{\circ} \mathrm{C}$;

- Product mass value of $12 \mathrm{~kg}$.

The effect of the ambient temperature (intended as the air temperature surrounding the external enclosure) on the performance of the refrigerator was not varied. Indeed, the purpose of this work is to investigate the possibility of achieving running cost saving of cabinet refrigerator placed in rooms where the ambient temperature does not change remarkably, such as temperature-controlled rooms.

The results of the experimental tests were discussed in a previous paper (Maiorino et al., 2019), in which a detailed explanation of the experimental procedure can be found. In detail, the tests labelled as Hyst1, Hyst2, and Hyst3 are considered in this work to model the cyclic operations of the refrigerator. The previous work included a comprehensive analysis of the temperature distribution inside the refrigerator at different conditions that verified more appropriate conservation for the product with PCM than without it at different hysteresis. Hence, the temperature behaviour of the refrigerator is not considered in this work. The data used to model the cyclic operations of the refrigerator with PCM and without are presented in Tab. 1. $\Delta_{\exp }$ represents the hysteresis values used during the experimental tests, whereas $\tau^{\mathrm{ON}}$ and $\tau^{\mathrm{OFF}}$ are the $\mathrm{ON}$ and OFF time of the compressor during a working cycle, respectively.

The power absorbed $(\mathrm{P})$ by the compressor of the refrigerator system showed in Tab. 1 is evaluated as an average over the ON time (Maiorino et al., 2019).

\section{Optimisation methodology}

\subsection{Problem definition}

From Tab. 1, it is evident that the introduction of the PCM leads to increase both the ON and OFF time of the refrigerator, especially for higher hysteresis values. Nevertheless, the power absorbed by the compressor increases as the hysteresis value increases. The latter led to a rise in the daily energy consumption of the refrigerator with PCM up to $6.5 \%$. The experimental tests showed an increase in energy consumption of the refrigerator with PCM, especially at higher hysteresis. On the other hand, the significant increase of the OFF time when PCM is used offers a more flexible system that can be exploited, adapting its working scheduling to a TOU electricity tariff to reduce the running costs of such a system. The latter can be achieved by adjusting the working periods of the compressor of the refrigerator (ON time) to the off-peak periods of the TOU electricity tariff when the electricity 
cost is lower. Then, the energy stored in the PCM can be used to keep the temperature inside the cabinet of the refrigerator within the desired range without switching on the compressor during the peak periods of the TOU electricity tariff. Hence, it is needed to reduce the operations of the refrigerator during peak periods as much as possible.

The hysteresis is a crucial parameter to achieve this objective since it has a substantial impact on both energy consumption and OFF time. Its value should be selected to ensure that the OFF time of the compressor is long enough to avoid the switching on of the system during peak periods when the electricity cost is higher. Furthermore, the hysteresis also affects the ON time of the system, and it should be considered in the optimal scheduling problem. The aim to reduce the running costs of the refrigerator system can be addressed as an optimization problem with integer variables where the objective function is represented by the overall operative costs of the system over a pre-defined time horizon. The decision variables of the optimization problem are represented by the scheduled hysteresis over the pre-defined time horizon, which affect the cyclic operations of the system and its energy consumption.

\subsection{Objective function}

To solve the optimisation problem, the pre-defined time horizon $(H)$ is divided into different time-steps $(\delta \tau)$. Hence, the running cost over a pre-defined time horizon $\left(c_{H}\right)$ can be expressed as presented in Eq. 1.

$$
c_{H}=\sum_{i=1}^{H} c_{i} * P_{i}\left(\Delta_{i}\right) * S_{i} * \delta \tau=\sum_{i=1}^{H} c_{i} * E C_{i}
$$

Referred to the $\mathrm{i}$-th time step, $c_{i}$ is the unit energy cost, $P_{i}$ is the average power absorbed by the compressor, $\Delta_{i}$ is the value of the hysteresis, $s_{i}$ is the state of the compressor and $E C_{i}$ is the electric energy absorbed by the compressor. The state of the compressor indicates the status of the system, being 1 if the compressor is $\mathrm{ON}$ and 0 if it is OFF. The optimisation problem aims to minimise Eq. 1, which represents its objective function, and therefore, it can be formulated as showed in Eq. 2.

$$
\min _{\Delta_{i} \in \Delta_{\exp }} c_{H}=\sum_{i=1}^{H} c_{i} * P_{i}\left(\Delta_{i}\right) * s_{i} * \delta \tau=\sum_{i=1}^{H} c_{i} * E C_{i}
$$

$\Delta_{\text {exp }}$ is the set of hysteresis values that were used during the experimental tests, included in Tab. 1. The overall running cost strongly depends on the unit energy $\operatorname{cost} c$ and the power absorbed by the compressor $P$. The former can be considered as a parameter since it is an external variable that cannot be controlled. Indeed, the energy cost can vary significantly depending on the country, and the supplied considered.

On the other hand, the power absorbed by the refrigerator represents the total refrigeration system consumption and depends on the hysteresis control, as highlighted by the experimental results (Tab. 1). Additionally, the hysteresis control also affects the ON and OFF time of the compressor, and therefore, the energy consumption. Hence, the hysteresis can be considered as the decision variable 
of the optimisation problem. In detail, it is necessary to find the optimal scheduling of hysteresis values that define the optimal working schedule of the refrigerator, considering the ON and OFF time of the compressor. A hysteresis value $\left(\Delta_{i}\right)$ should be defined for each time-step to identify the power absorbed by the compressor at the $\mathrm{i}$-th time-step $\left(P_{i}\right)$. The array of hysteresis values (built as a function of the time) depends on the array of scheduled hysteresis $\left(\overline{\Delta^{S}}\right)$ over the pre-defined time horizon, as defined by Eq. 3 .

$$
\Delta_{i}=\bar{\Delta}\left(\overline{\Delta^{s}}\right)=\Delta_{i}\left(\Delta_{j}^{S}\right), j=1,2, \ldots, n \text { and } i=1,2, \ldots, H
$$

In Eq. 3, $n$ represents the number of scheduled hysteresis, that is the size of $\overline{\Delta^{s}}$, and $\Delta_{j}^{S}$ is the j-th scheduled hysteresis. The array of scheduled hysteresis $\overline{\Delta^{s}}$ represents the entire domain of decision variables.

\subsection{Constraints}

The objective function is subjected to different constraints that are related to the cyclic operations of the refrigeration system, in terms of $\mathrm{ON}$ and OFF time. First, it is needed to define the starting times of the compressor over the defined time-horizon $\left(\tau_{j}^{S S}\right)$. An initial condition must be defined as the first starting time of the compressor $\left(\tau_{1}^{S S}\right)$, as shown in Eq. 4. Then, each starting time of the compressor $\left(\tau_{j}^{S S}\right)$ over the pre-defined time horizon can be computed according to Eq. 5 .

$$
\begin{gathered}
\tau_{1}^{S S}=0 \\
\tau_{j}^{S S}=\tau_{j-1}^{S S}+\tau^{O N}\left(\Delta_{j-1}^{S}\right)+\tau^{O F F}\left(\Delta_{j-1}^{S}\right), \forall j \in[2, \ldots, n]
\end{gathered}
$$

The constraint expressed by Eq. 5 defines that a new cycle cannot start before the previous one is finished. It means that the next starting time of the compressor must be equal to the previous starting time $\left(\tau_{j-1}^{S S}\right)$ plus the cycle time of the system, defined as the sum of the ON time $\left(\tau^{O N}\right)$ and the OFF time $\left(\tau^{O F F}\right)$ of the compressor. Both ON and OFF times depend on the scheduled hysteresis value chosen for the previous cycle $\left(\Delta_{j-1}^{S}\right)$. Some constraints regarding the state of the compressor are also needed, and they are presented below.

$$
\begin{gathered}
s_{i} \in[0,1], \forall i \in[1, \ldots, H] \\
\sum_{i=\tau_{j}^{S S}}^{\tau^{O N}\left(\Delta_{j}^{S}\right)} s_{i}=\tau^{O N}\left(\Delta_{j}^{S}\right), \forall j \in[1, \ldots, n] \\
\sum_{i=\tau_{j}^{S S}+\tau^{O N}\left(\Delta_{j}^{S}\right)+1} s_{i}=0, \forall j \in[1, \ldots, n]
\end{gathered}
$$


Eq. 6 expresses the binary conditions for the variables $s_{i}$. It means that the state of the compressor can assume only two integer values: 1 or 0 . Then, it is necessary to establish that the state of the compressor must be equal to 1 for each time-step $i$ during the ON period of each cycle $j$ (Eq. 7). In this way, the state of the compressor must be equal to 0 for each time-step $i$ during the OFF period of each cycle $j$ (Eq. 8). These constraints define the array of the states of the compressor for each timestep as a function of the scheduled hysteresis $\Delta_{j}^{S}$. This array directly affects the objective function of the optimisation problem.

To guarantee that, for each time-step, the hysteresis can assume only the values used during the experimental tests $\left(\Delta_{\text {exp }}\right)$, a constraint expressed by Eq. 9 was introduced.

$$
\Delta_{i} \in[1,2,3], \forall i \in[1, \ldots, H]
$$

In Eq. 10, the set of constraints needed to identify the hysteresis for each time step are shown. This set of equations ensures that the hysteresis does not change until the working cycle, defined as a function of the j-th scheduled hysteresis, ends. It means that $\Delta$ must be equal to $\Delta_{j}^{S}$ from $\tau_{j}^{S S}$ to $\tau^{O N}\left(\Delta_{j}^{S}\right)+\tau^{O F F}\left(\Delta_{j}^{S}\right)$. With Eq. 10, the array of hysteresis as a function of time $(\bar{\Delta})$ is defined.

$$
\begin{gathered}
\Delta_{\tau_{j}^{s S}}=\Delta_{j}^{S} \\
\Delta_{\tau_{j}^{S S}+1}=\Delta_{j}^{S} \\
\vdots \\
\Delta_{\tau_{j}^{S S}+\tau^{O N}\left(\Delta_{j}^{S}\right)}=\Delta_{j}^{S} \quad, \forall j \in[1, \ldots, n] \\
\Delta_{\tau_{j}^{S S}+\tau^{O N}\left(\Delta_{j}^{S}\right)+1}=\Delta_{j}^{S} \\
\vdots \\
\Delta_{\tau_{j}^{S S}+\tau^{O N}\left(\Delta_{j}^{S}\right)+\tau^{O F F}\left(\Delta_{j}^{S}\right)}=\Delta_{j}^{S}
\end{gathered}
$$

The remaining variables of the mathematical problem are defined in Eq. 11-15, such as the electricity cost profile (Eq. 11), the power absorbed by the compressor for each time-step as a function of the hysteresis (Eq. 12), the time-step (Eq. 13), the time horizon (Eq. 14) and the number of scheduled hysteresis (Eq. 15).

$$
\begin{gathered}
\bar{c}=\left[c_{1}, c_{2}, c_{3}, \ldots, c_{H-1}, c_{H}\right] \\
\bar{P}(\bar{\Delta})=\left[P_{1}\left(\Delta_{1}\right), P_{2}\left(\Delta_{2}\right), P_{3}\left(\Delta_{3}\right), \ldots, P_{H-1}\left(\Delta_{H-1}\right), P_{H}\left(\Delta_{H}\right)\right] \\
\delta \tau=1 \\
H=H_{\text {set }} \\
n=n_{\text {set }}
\end{gathered}
$$

These last parameters are defined a priori $\left(H_{\text {set }}\right.$ and $n_{\text {set }}$, respectively). The electricity cost profile depends on the specific case study. The power absorbed by the compressor was measured during the experimental tests, and the possible values are reported in Tab. 1. The time-step was fixed to $1 s$ to have a better representation of the cyclic operations of the system. 
The optimisation problem was solved by the Simulated Annealing (SA) method (see Appendix A) that allowed identifying the optimal scheduling of the refrigerator for different case studies. This meta-heuristic technique was used since it is straightforward to code and implement, and it is a wellestablished technique in the field of optimisation problems. However, different methods, such as genetic algorithms, particle swarm or ant colony method, could be used to solve the optimisation problem presented above. Still, the comparison of these methods is not the aim of this work.

\section{Results and discussion}

\subsection{Case studies}

The possibility of achieving running cost savings installing PCM in a cabinet refrigerator was investigated considering several case studies. These case studies are referred to different TOU electricity tariffs, which can be chosen by a domestic user. The selected TOU electricity tariffs used to test the optimisation method described in Section 3 are shown in Tab. 2.

The selected TOU electricity tariffs can be divided into two categories: tariffs in which the unit energy cost can have two values (2-TOU tariffs) and other with three possibilities (3-TOU tariffs). The variation of the unit energy cost in both categories depends on the time usage of electric energy. There is a time range in which the unit energy cost is higher (peak periods) since the demand is higher as well. Then, the unit energy cost decreases when the demand also decreases (off-peak and super off-peak periods). Different TOU tariffs found in supplier companies of three countries (Spain, Italy, and France) were studied to assess the effect of the variation of the unit energy cost. A particular tariff is also considered for France, which is characterised by a 2-TOU tariff by day with different peak and off-peak prices according to the day. Indeed, these costs are different for blue days (FR2bd), white days (FR2wd), and red days (FR2rd), which are defined by the energy service provider.

The case studies described above were investigated by identifying the optimal daily scheduling of the refrigerator with PCM for each of them. The energy consumption of the optimal cyclic operations of the system with PCM was then compared to its normal operation without PCM and considering three different fixed hysteresis. After, the optimisation routine was also performed, extending the time horizon to the week. In this case, only 3-TOU tariffs were considered since the TOU distribution can vary day by day over the week.

\subsection{Daily optimisation}

The introduction of the PCM allows the extension of the OFF time of the refrigerator since it stores thermal energy. Therefore, PCMs could reduce the working time of the system during the peak period, and consequently, the overall energy costs.

Fig. 1 summarises the results of the optimisation method for the different case studies of 2-TOU tariffs, showing a comparison of the daily running costs of the refrigerator with PCM (optPCM) and without it (noPCM 1, noPCM 2, noPCM 3).

A reduction of the daily running costs of the system can be achieved optimising the hysteresis scheduling of the refrigerator with PCM for almost all the selected cases. The magnitude of the reduction depends on the specific case study. The percentages above the green bars in Fig. 1 represents the variation of the daily running cost between the best working operations of the refrigerator without PCM (fixed hysteresis) and the optimised working operations of the refrigerator with PCM. In detail, considering a country like Spain (SP2a, SP2b, SP2c, and SP2d), a decrease of the daily running costs ranging from $20.4 \%$ to $22.5 \%$ can be achieved according to the energy service provider. In France, this benefit is not so evident since the reduction of the daily running costs is equal 
to $1.9 \%$ for the standard 2-TOU tariffs (FR2). Focusing on the $3 \times 2$ TOU tariff, it can be noticed that the PCM has a negative effect during blue days (FR2bd) also after the scheduling optimisation, with a running cost increase of $0.8 \%$. In contrast, it shows a slight improvement during white days (FR2wd). On the other hand, the scheduling optimisation with PCM allows achieving a reduction of the daily running costs of $35.0 \%$ during red days. The economic benefits observed for Spain and France are not evidenced in the Italian case study, where the introduction of PCM leads to an increase in the daily running costs of the refrigerator despite the scheduling optimisation. To find out the motivation of these different results, in Fig. 2, the daily cost saving dependence on the ratio between the off-peak and peak cost is shown. Indeed, it was expected that the economic benefit of the optimisation scheduling with PCM could be related to this ratio.

From Fig. 2, it can be noticed that the daily running cost reduction decreases as the ratio between the off-peak and peak cost increases. It means that if the unit energy cost during off-peak periods is close to peak period, an economic benefit that can be achieved by optimising the working operations of the refrigerator with PCM is lower or even not existing, as it happens for the Italian case study. The daily running cost savings shown in Fig. 2 are calculated comparing the results of the optimised working operations of the refrigerator with PCM and the working operations of the refrigerator without PCM with three different fixed hysteresis controls (Hyst1, Hyst2, and Hyst3). Considering a specific off-peak vs peak cost ratio, the running cost saving is higher if the refrigerator is controlled with a hysteresis value equal to $1 K$ (green line in Fig. 2). In contrast, it is reduced if the refrigerator without PCM is controlled with hysteresis values equal to 2 or $3 K$ (light blue and yellow lines, respectively). However, this figure can be helpful in understanding the upper limit of the ratio between the off-peak and peak cost to ensure achieving a running cost saving by scheduling optimisation of the refrigerator with PCM. For the investigated case studies, this upper limit is around $80 \%$, and it means that the off-peak unit energy cost must be less than $80 \%$ of the peak unit energy cost to ensure an economic benefit.

To complete the daily analysis, the results of the scheduling optimisation with 3-TOU tariffs are reported in Fig. 3.

From Fig. 3, one can see that the daily running costs are reduced for the Spanish case study. Indeed, the scheduling optimisation of the refrigerator with PCM leads to a reduction of the daily running costs of $20.8 \%$ and $5.0 \%$ for the two energy service providers investigated (SP3a and SP3b, respectively). On the other hand, the Italian scenario with a 3-TOU tariff shows that the PCM increases the daily running costs of the system $(+4.1 \%)$, despite the optimised scheduling carried out. These results are comparable to those presented for the 2-TOU tariffs. It can be concluded that the differences between the different periods (peak, off-peak, and super off-peak) are very slight in Italy compared to the Spanish ones. Therefore, the scheduling optimisation of the refrigerator with PCM does not achieve an economic benefit in this country. However, these results were obtained excluding the effect of ambient temperature on the energy performance and the impact of door openings. Indeed, these two factors could lead to different conclusions, especially considering other applications, where the effect of the ambient temperature cannot be neglected, such as mobile systems.

\subsection{Weekly optimisation}

Some of the TOU tariffs used to test the optimisation procedure are characterised by a variation of the cost profile day by day along a week. Indeed, the cost profile during a weekday (from Monday to Friday) could be different from the cost profile during the weekend (Saturday and Sunday). In detail, most of the 3-TOU tariffs are characterised by these differences between weekdays and the weekend. Hence, the daily scheduling optimisation could not be sufficient to represent the actual 
working operations of the refrigerator. For this reason, the optimisation procedure was also performed considering a weekly time horizon, and the results are shown in Fig. 4.

From Fig. 4, it can be evidenced that the optimisation routine provides different power profiles for the different case studies, that are related to different hysteresis scheduling, as a response to different cost profiles. However, it is worth to notice that the optimisation routine can minimise the working time of the refrigerator during peak periods over a week for all three case studies. Furthermore, focusing on the Italian case study (IT3), it is evident that the differences between each TOU are minimal. Therefore, a minor effect of the scheduling optimisation is expected, as explained in Section 4.1. On the other hand, Spain presents a more significant change in the unit energy cost between one TOU from another, especially considering the first energy service provider (SP3a).

Fig. 5 summarises the results of the optimisation method applied with a weekly time horizon for the different case studies of 3-TOU tariffs showing a comparison of the weekly running costs of the refrigerator with PCM (optPCM) and without it (noPCM 1, noPCM 2, noPCM 3).

The results are similar to those obtained with a daily optimisation, in terms of percentage costsaving (reported above the green bars), showing an economic benefit for the Spanish case studies up to $-14.0 \%$ and an increase of the running costs of the refrigerator for the Italian case study $(+5.9 \%)$. Indeed, the same considerations made for the daily optimisation about are still applicable to explain the weekly optimisation results.

\section{Conclusions}

This work proposes an optimisation algorithm to control the operations of a cabinet refrigerator equipped with PCM attached to a bare tube evaporator, which in principle, cannot provide economic benefits with a simple operation because of higher electric energy absorption. The selection of the hysteresis offers flexibility.

The optimisation problem aims to minimise the overall running cost over the pre-defined time horizon finding the optimal scheduling of hysteresis values that defines the optimal working schedule of the refrigerator. The Simulated Annealing (SA) method was used to solve the proposed optimisation problem since it is a straightforward technique that can be efficiently coded and implemented. The individuation of the optimal algorithm is not the main scope of this paper, and therefore, it is left to future works. Nine 2-TOU tariffs (peak and off-peak) and three 3-TOU tariffs (peak, off-peak, super off-peak) from three European countries (Spain, Italy, and France) have been selected for the analysis.

Refrigerator operating at different hysteresis without PCM and the optimised operations of the refrigerator with PCM are simulated and compared. Considering daily operations, most of the selected 2-TOU case studies provide economic benefits compared to the best option without PCM, except for the Italian electricity supplier. If the off-peak unit energy cost is $80 \%$ lower than that of the peak unit energy cost, there is an economic benefit for the proposed controlled system. The highest benefit for 2-TOU tariffs is found for red days in France, followed by the four Spanish electricity suppliers. The presence of the super off-peak period in 3-TOU tariffs does not result in higher economic benefits.

The analysis of the weekly time horizon, which considers the differences between weekdays and weekends, provides complimentary conclusions to this analysis because the differences between both periods are noticeable. The optimisation routine can minimise the working time of the refrigerator during peak periods over a week for all proposed cases, in comparison with the daily time horizon. 
Spanish electricity suppliers maintain the economic benefit while the opposite results for the Italian case.

This work has proved the benefit of the combination of optimisation methods in thermal systems that includes PCM even in the cases which benefit is not apparent. Further investigations could also consider the effect of the surrounding air temperature on the average power absorbed by the compressor, as well as the impact of door openings on the cyclic operations of the refrigerator. These effects could make favourable the application of PCM also in those cases where it seems that there is no advantage in using it, such as in Italy.

\section{Acknowledgements}

Adrián Mota-Babiloni would like to acknowledge the funding received from the Spanish State Research Agency through the "Juan de la Cierva - Formación 2016" postdoctoral grant (FJCI-201628324) and from the Universitat Jaume I though its Research Stay Grants (E-2017-30).

\section{APPENDIX}

\section{A. The Simulated Annealing method}

The Simulated Annealing (SA) method was used to solve the optimisation problem described in the previous subsections. The SA is a meta-heuristic technique based on the Metropolis algorithm, which was first presented in 1953 (Metropolis et al., 1953). Then, (Kirkpatrick et al., 1983) applied for the first time this algorithm to solve an optimisation problem in 1983. The SA method was inspired by the step-by-step cooling process of annealing metal after it is heated to the melting point. At high temperature, the atoms of metal can arbitrarily move since they have high energy. When the cooling process starts, the metal starts to crystallize, and the energy of the atoms decreases going towards the minimum state of energy. At the end of the cooling process, the minimum state of energy is reached.

The SA optimisation method uses a stochastic approach to search for and move to new solutions, called neighbourhood solutions. These solutions are analogous to the different states of energy during the annealing process of metal. Hence, starting from an initial solution (initial state of energy) at an initial temperature $\left(T_{0}\right)$, a random perturbation is imposed to find a new neighbourhood solution (another possible state of energy). The initial and the neighbourhood solutions are represented by two different arrays of scheduled hysteresis $\Delta^{S}$. Then, the difference in the energy between the two solutions $\left(\triangle E=E_{2}-E_{1}\right)$ is computed. If the energy in the previous state $\left(E_{1}\right)$ is lower than the new one $\left(E_{2}\right)$, the new solution is accepted according to a probability function that depends on the present temperature $(T)$ of the process. On the other hand, if the energy in the previous state is higher than the new one, the new solution is always accepted. The energy of each solution is represented by the cost function $c_{H}$. The latter is calculated by computing $\overline{\tau^{S S}}, \bar{S}, \bar{\Delta}$ and $\bar{P}$ according to the constraints of the optimisation problem. After, the search for new solutions can continue decreasing the temperature according to a defined cooling schedule until the stopping conditions are reached. Stopping conditions can be defined by the final temperature of the process $\left(T_{\text {end }}\right)$ or by the maximum number of iterations. For each temperature step, different iterations $(s t)$ can be performed at the same temperature to 
explore the entire space of solutions. Any functions can represent the cooling schedule as a function of a defined cooling rate $(\alpha)$. In this work, a geometric temperature decrease was applied with a cooling rate of 0.99 . The temperatures used in the SA method are dummy variables which allow simulating the cooling process of annealing metal. In detail, the initial temperature $\left(T_{0}\right)$ can be defined according to the chosen acceptance probability of the first neighbourhood solution whereas the final temperature $\left(T_{\text {end }}\right)$ can be defined according to the chosen acceptance probability of the last found solution. The acceptance probability (A) of a new solution is defined by the following equation:

$$
A=e^{-\frac{\Delta E}{T}}
$$

In this work, the acceptance probabilities for the first and the last solution were set to 0.95 and 0.05 , respectively. Hence, the initial and final temperatures were calculated as follows:

$$
\begin{gathered}
T_{0}=-\frac{E_{2}-E_{1}}{\ln 0.95} \\
T_{\text {end }}=-\frac{E_{2}-E_{1}}{\ln 0.05}
\end{gathered}
$$

The SA algorithm to solve the optimisation problem described in sub-sections 3.1-3.3 was implemented using MATLAB, and the corresponding flow-chart is shown in Fig. A.1. The inputs of the algorithm are the unit energy cost profile, the SA parameters (the initial and the final temperature, the cooling rate and the number of iterations per each temperature step), the optimisation time horizon and the time-step. The running cost, the optimised scheduling of hysteresis values and the profile of the power absorbed by the compressor over the pre-defined time horizon represent the outputs of the entire procedure.

\section{REFERENCES}

Azzouz, K., Leducq, D., Gobin, D., 2009. Enhancing the performance of household refrigerators with latent heat storage: An experimental investigation. Int. J. Refrig. 32, 1634-1644. https://doi.org/10.1016/J.IJREFRIG.2009.03.012

Barzin, R., Chen, J.J.J., Young, B.R., Farid, M.M., 2015. Peak load shifting with energy storage and price-based control system. Energy 92, 505-514. https://doi.org/10.1016/J.ENERGY.2015.05.144

Bergaentzlé, C., Jensen, I.G., Skytte, K., Olsen, O.J., 2019. Electricity grid tariffs as a tool for flexible energy systems: A Danish case study. Energy Policy 126, 12-21. https://doi.org/10.1016/J.ENPOL.2018.11.021

Bista, S., Hosseini, S.E., Owens, E., Phillips, G., 2018. Performance improvement and energy consumption reduction in refrigeration systems using phase change material (PCM). Appl. Therm. Eng. 142, 723-735. https://doi.org/10.1016/J.APPLTHERMALENG.2018.07.068

Cofré-Toledo, J., Vasco, D.A., Isaza-Roldán, C.A., Tangarife, J.A., 2018. Evaluation of an integrated household refrigerator evaporator with two eutectic phase-change materials. Int. J. Refrig. 93, 29-37. https://doi.org/10.1016/J.IJREFRIG.2018.06.003

Coulomb, D., Dupont, J.-L., Pichard, A., 2015. The Role of Refrigeration in the Global Economy. 29th Informatory Note on Refrigeration Technologies. Paris (France).

Coulomb, D., Dupont, J.-L.L., Marlet, V., Morlet, V., 2017. The impact of the refrigeration sector on the climate change. 35th Informatory Note on Refrigeration Technologies. Paris (France).

Dlamini, N.G., Cromieres, F., 2012. Implementing peak load reduction algorithms for household electrical appliances. Energy Policy 44, 280-290. https://doi.org/10.1016/J.ENPOL.2012.01.051

Elarem, R., Mellouli, S., Abhilash, E., Jemni, A., 2017. Performance analysis of a household refrigerator integrating a 


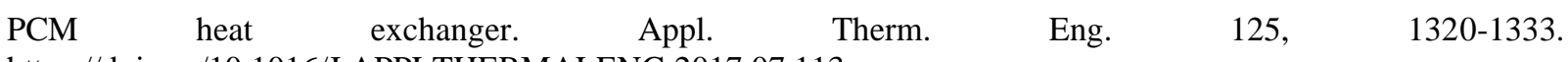
https://doi.org/10.1016/J.APPLTHERMALENG.2017.07.113

Hovgaard, T.G., Larsen, L.F.S., Edlund, K., Jørgensen, J.B., 2012. Model predictive control technologies for efficient and flexible power consumption in refrigeration systems. Energy 44, 105-116. https://doi.org/10.1016/J.ENERGY.2011.12.007

Kirkpatrick, S., Gelatt, C.D., Vecchi, M.P., 1983. Optimization by Simulated Annealing. Science (80-. ). 220, 671-680. https://doi.org/10.1126/science.220.4598.671

Li, S.-F., Liu, Z., Wang, X.-J., 2019. A comprehensive review on positive cold energy storage technologies and applications in air conditioning with phase change materials. Appl. Energy 255, 113667. https://doi.org/https://doi.org/10.1016/j.apenergy.2019.113667

Liu, Z., Zhao, D., Wang, Q., Chi, Y., Zhang, L., 2017. Performance study on air-cooled household refrigerator with cold storage phase change materials. Int. J. Refrig. 79, 130-142. https://doi.org/10.1016/J.IJREFRIG.2017.04.009

Maiorino, A., Del Duca, M.G., Mota-Babiloni, A., Greco, A., Aprea, C., 2019. The thermal performances of a refrigerator incorporating a phase change material. Int. J. Refrig. https://doi.org/10.1016/j.ijrefrig.2019.02.005

Mastani Joybari, M., Haghighat, F., Moffat, J., Sra, P., 2015. Heat and cold storage using phase change materials in domestic refrigeration systems: The state-of-the-art review. Energy Build. 106, 111-124. https://doi.org/10.1016/j.enbuild.2015.06.016

McNeil, M.A., Karali, N., Letschert, V., 2019. Forecasting Indonesia's electricity load through 2030 and peak demand reductions from appliance and lighting efficiency. Energy Sustain. Dev. 49, 65-77. https://doi.org/10.1016/J.ESD.2019.01.001

Metropolis, N., Rosenbluth, A.W., Rosenbluth, M.N., Teller, A.H., Teller, E., 1953. Equation of State Calculations by Fast Computing Machines. J. Chem. Phys. 21, 1087-1092. https://doi.org/10.1063/1.1699114

Purohit, N., Dasgupta, M.S., 2020. Thermal storage material enhanced refrigerated display cabinet. Mater. Today Proc. https://doi.org/https://doi.org/10.1016/j.matpr.2019.12.210

Schné, T., Jaskó, S., Simon, G., 2018. Embeddable adaptive model predictive refrigerator control for cost-efficient and sustainable operation. J. Clean. Prod. 190, 496-507. https://doi.org/10.1016/J.JCLEPRO.2018.04.137

Wang, C., He, Z., Wennerstern, R., Sun, Q., 2017. Evaluation on Performance of a Phase Change Material Based Cold Storage House. Energy Procedia 105, 3947-3952. https://doi.org/10.1016/J.EGYPRO.2017.03.820

Wang, Y., Lin, H., Liu, Y., Sun, Q., Wennersten, R., 2018. Management of household electricity consumption under price-based demand response scheme. J. Clean. Prod. 204, 926-938. https://doi.org/10.1016/J.JCLEPRO.2018.09.019

Yan, X., Ozturk, Y., Hu, Z., Song, Y., 2018. A review on price-driven residential demand response. Renew. Sustain. Energy Rev. 96, 411-419. https://doi.org/10.1016/J.RSER.2018.08.003

Zehir, M.A., Bagriyanik, M., 2012. Demand Side Management by controlling refrigerators and its effects on consumers. Energy Convers. Manag. 64, 238-244. https://doi.org/10.1016/J.ENCONMAN.2012.05.012 
